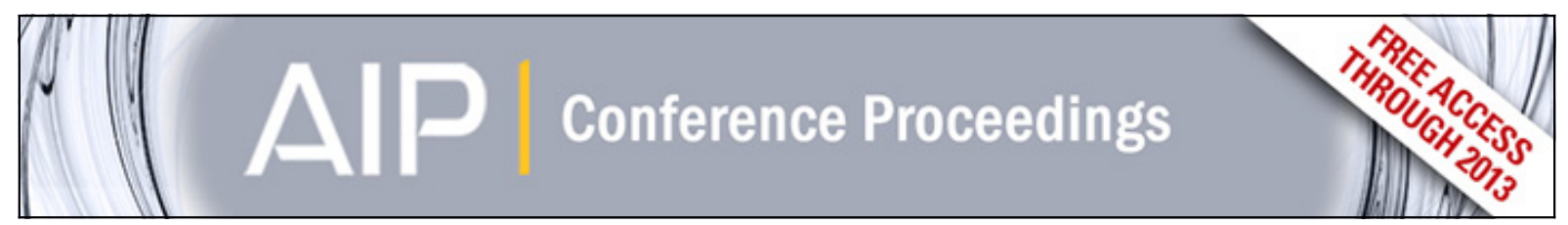

Non Kolmogorov Probability Models Outside Quantum Mechanics

Luigi Accardi

Citation: AIP Conference Proceedings 1101, 3 (2009); doi: 10.1063/1.3109969

View online: http://dx.doi.org/10.1063/1.3109969

View Table of Contents: http://scitation.aip.org/content/aip/proceeding/aipcp/1101?ver=pdfcov

Published by the AIP Publishing 


\title{
Non Kolmogorov Probability Models Outside Quantum Mechanics
}

\author{
Luigi Accardi \\ Centro Vito Volterra, \\ Università degli Studi di Roma "Tor Vergata", \\ 00133 Roma, Italy
}

\begin{abstract}
This paper is devoted to analysis of main conceptual problems in the interpretation of QM: reality, locality, determinism, physical state, Heisenberg principle, "deterministic" and "exact" theories, laws of chance, notion of event, statistical invariants, adaptive realism, EPR correlations and, finally, the EPR-chameleon experiment.
\end{abstract}

Keywords: quantum probability, adaptive systems, statistical invariants

PACS: 02.50.-r, 04.20.Cv, 03.65.Ta, 03.65.Ud

\section{WHICH CONCEPTS WERE REVOLUTIONIZED BY QM?}

Since the early times of the theory it was clear to everybody that QM was a conceptual revolution, however the question:

which concepts were revolutionized by $Q M$ ?

received different answers.

In the early stage of the theory, i.e. in the period 1926-27, the dominating opinion was that the main conceptual revolution had to do with

(I) determinism (or better, its rejection)

Heisenberg indeterminacy principle was considered as a physical evidence of an intrinsic quantum indeterminism and the statistical interpretation of the wave function gave further support to this idea.

\section{A digression on determinism}

The terms "determinism" and "physical state" have undergone a parallel evolution which has been analyzed in the paper [1]. This evolution has created some confusions in the literature due to the fact that the dynamical law of quantum theory, Shrödinger equation, being the solution of a Cauchy initial value problem, is perfectly deterministic. The root of these confusions is in the distinction between "deterministic" and "exact" theories. The former term refers to the dynamical evolution and means that, knowing the past, one can exactly predict the future; the latter refers to the fact that the theory does not include probabilistic assumptions (in the proper sense, specified below).

Classical Hamiltonian dynamics is the prototype of exact, deterministic reversible theory in the newtonian sense.

Hereditary dynamics, describing evolutions depending on the whole past history of a system and not only on the present state, provide examples of non Newtonian, non reversible determinism.

Classical thermodynamics is an example of exact theory, but one would not call it a deterministic theory.

Markov processes provide examples of non exact (statistical) deterministic, generically irreversible, theories.

Classical statistical mechanics and quantum mechanics are examples of (Newtonian) deterministic, reversible statistical theories.

However classical statistical mechanics (CSM) has an underlying exact theory, which is classical Hamiltonian mechanics (CHM): the difference between the two is only in the notion of state. In CHM the states are the points of the phase space, in (standard) CSM the states are the measures on the phase space which are absolutely continuous with respect to the Lebesgue measure (on the whole phase space or on some energy surface of it).

Identifying the points of the phase space with the corresponding Dirac measures one obtains a formal inclusion of CHM within CSM. This is a general for classical theories: by allowing Dirac measures among admissible states, every 
exact theory can be embedded in a statistical theory. A statistical theory is called properly statistical is it includes probability measures with support not reduced to a single point. Quantum theory is an extreme case of such theories since in it no state is delta-like for any observable.

The possibility to construct an underlying exact, deterministic, reversible physical theory, playing for quantum mechanics the same role played by CHM for CSM, is the core of the line of research dealing with hidden variables.

There are several inequivalent ways to formulate this problem in a precise mathematical language and the conclusion on the existence or non existence of such an underlying theory strongly depends on the formulation.

A discussion of some of the best known proposals in this direction is contained in [2].

\section{Back to the different answers}

In the period 1928-64 the dominating opinion was that the main conceptual revolution had to do with

(II) reality (or better the renounce of the old naive idea people had of this notion).

According to this point of view, QM implies a new notion of reality (virtual reality) and the main experimental support to this thesis was looked for in the two slit type experiments which lead to two slit type inequalities.

In the period 1964-1979, starting with Bell's paper, the dominating paradigm became that the main conceptual revolution had to do with:

(III) locality (or better, its rejection)

Bell revived the idea, already present in the Bohr-Einstein debate during the 1928 Solvay conference, that the combination of QM with the usual notion of reality implied nonlocality, in the sense of action at distance.

The main experimental support to this thesis was looked for into the EPR type experiments which lead to Bell's type inequalities.

Since the experiments seemed to confirm the validity of QM and the conservation laws between distant particles were interpreted as an experimental support of reality, many people concluded that in nature there should be an intrinsic nonlocality, also-called quantum nonlocality because the problem doesn't arise in classical physics.

\section{Quantum Probability}

The thesis of QP is that the main conceptual revolution implied by QM has to do with

\section{(IV) the laws of chance}

The elder two slit type inequalities were proved to have the same conceptual content as Bell's type inequalities: necessary conditions for the existence of a classical (Kolmogorov) probabilistic model.

The paradigmatic experiments, both two slit type or EPR type, were interpreted as evidence that there exist families of statistical data, collected in rather similar physical situations and relative to similar systems, which cannot be described within a single classical probabilistic model (inadequacy of the old) but are very efficiently described by a single quantum probabilistic model (greater power of the new).

Nowadays everybody gives this conclusion for granted and few remember that it took decades of struggle and isolation to make it accepted.

A natural implication from the above analysis was that, just like geometry did in the 19-th century, probability theory in the 20-th century had better accept the idea of a multiplicity of inequivalent mathematical models for the laws of chance.

In order to make this thesis a solid scientific theory, several important questions had to be answered. Moreover, as frequently happens in science, often the solution of a problem creates a new one.

That is the reason why it took about 30 years, starting from 1979, to develop a coherent quantum probabilistic approach to the foundations of quantum mechanics. This process went through several steps, related to the successive clarification of different problems. In the following I will, very quickly and schematically, describe the main among these ones. I will not give bibliographical references for this development: they can be found in the volumes of the proceedings of the Växjö conferences [3].

The period 1979-1981 was devoted to give a mathematical content to the historical analogy with geometry mentioned above.

The distinction among different mathematical models of space (geometries) was achieved by Gauss through the definition and computation of geometrical invariants. The main property of these objects, already noted by Gauss, was 
that they are experimentally measurable.

To define their probabilistic analogues, which is natural to call statistical invariants, was the first challenge QP had to meet in this period. The solution of this problem, expressed in terms of explicit computation of these invariants in some special but important and physically meaningful cases, raised the following new question.

The statistical invariants allow to solve problems of the form: given two different mathematical models of the laws of chance (probabilistic models) how to distinguish among them?

However, just because the existence of different models to be compared is their starting point, the statistical invariants cannot explain the emergence of the specific features of different models from physically reasonable requirements.

The period 1981-1993 was devoted to the clarification of this problem. The main result was the deduction of the QM probabilistic model from a single postulate which captures the conceptual essence (i.e. independent of specific observables) of Heisenberg indeterminacy principle:

\section{there exist pairs of incompatible observables.}

Surprising as it may seem, all the specific mathematical features of the QM mathematical model (including a generalization of the Hilbert space, the superposition principle, the Schrödinger equation, the theory of group representations, ... ) can be deduced from this qualitative assumption.

In addition to the usual quantum one, this assumption justifies the emergence of non (nontrivial) models which are neither classical nor quantum.

This result confirms the conviction that the role of axioms in a physical theory is to extract the essential physical requirements which are sufficient to justify the entire mathematical model.

Only when such a task has been achieved one can claim that the conceptual essence of a physical theory has been understood.

The period 1993-2001 was devoted to the clarification of the following problem: once accepted the existence of non classical statistics, which physical systems can produce non classical statistics? Can we build concrete examples of classical systems giving rise to non classical statistics? Can one reproduce the famous EPR correlations with deterministic, reversible, classical systems making totally independent local choices?

The period 2001-2008 was devoted to find the answers to these questions. The first step in this direction was the introduction of the locality and causality requirements in von Neumann's measurement theory.

This development was a key step to construct the first example of:

classical, deterministic, local, reversible dynamical system (thus without spurious external selections) exactly reproducing the EPR correlations.

This was done in the EPR-chameleon experiment which exploited the fact that local deformations of deterministic reversible trajectories give a simple intuitive explanation of Bell's type inequalities.

The theory underlying the EPR-chameleon experiment was completed in 2008 with the proof of the (essential) uniqueness of the the EPR-chameleon model in the following sense:

any classical, deterministic, local, reversible dynamical system exactly reproducing the EPR correlations must be a convex combination of EPR-chameleon models .

But a mathematical (rather complex) model can be considered to close a conceptual debate only to the extent that it inspires an intuitive and easily communicable picture of the difficulty and of its overcoming.

Thus one more challenge had to be overcome to complete the conceptual picture, one not having to do with mathematics but with intuition:

Can one give an intuitive justification of the emergence of non classical statistics, one that does not appeal to QM and that could be understood by an housewife?

The following section will shortly outline an answer to this question.

In conclusion: we have now understood that adaptive systems can produce non classical statistics. But adaptive systems are not peculiar to quantum theory: there are plenty of adaptive systems in classical contexts. Therefore for the future, i.e. after 2008 , it is natural to expect that the following program will play a relevant role in our understanding of the conceptual foundations of probability theory:

look for experimental data outside QM which justify the emergence of non Kolmogorov Probability models .

\section{WHY CHAMELEONS CAN PRODUCE NON CLASSICAL STATISTICS?}

Beyond the Heisenberg principle, which in its weak form asserts the existence of pairs of measurements which cannot be performed simultaneously on the same system, another important conceptual novelty introduced by quantum 
physics is the active role of the measurement operations, which has been repeatedly emphasized by Niels Bohr.

The quantum probabilistic approach pushes Bohr's point of view further by interpreting it as the statement that the properties of the objects should be thought as responses to interactions (i.e. measurements).

This means that:

- classical statistics is passive: we register what was there

- quantum statistics is active: we stimulate a response.

The chameleon metaphora illustrates the basic idea that:

- Classical systems are like balls in a box (basic example of CP).

- Quantum systems are like chameleons in a box (basic example of QP).

Out of the metaphora the problem stated at the end of the previous section can be rephrased as follows:

Can adaptive systems can produce non classical statistics?

The answer is yes! and in this section we illustrate this answer with a very simple example which has nothing to do with QM.

An ideal scheme of a measurement of a property $A$ is given by a black box (filter) that separates, either physically or in the perception of the experimenter, the systems that enjoy property $A$ from the others. We will identify property $A$ with the corresponding filter.

One can suppose that property $A$ is stable that is: any system that has passed the filter $A$ will pass an identical filter $A$ if meanwhile it has not been subject to other interactions.

In the black box two different physical process can take place. To explain this difference with a simple example we suppose that the system is a little bead and that property $A$ consists in the fact that the color of the bead is red. We then distinguish two cases:

(I) passive recording. The black box reads the color of the particle and doing so none of its properties is altered in a significant way (this is the point of view of classical probability)

(II) interaction and response. The black box asks the bead: "Is your color red? $\check{T}$ (in the following we will simply write: Red?) and the bead answers: Yes or No. Then it records in its memory this answer and, if it is Yes, it will give the same answer whenever asked again the same question (this illustrates the point of view of the quantum probability).

Good experimentalists are masters in the art of asking questions to nature and, in the following, the terms ask and answer should be interpreted in this sense.

Case (I) can be seen as a particular case of case (II) in the following way: a bead, to the question Red? , answers either always Yes or always $N o$ and in the former case it answers $N o$ for every other color (tertium non datur).

It is also clear that case (II) leaves much more freedom of choice to the bead: the rules stated so far do not exclude that, after having answered Yes to the question Red ? the bead can answer Yes' in case the next question is: Blue? (in this case tertium datur: we could say that quantum particle obey intuitionistic logic).

With contemporary technology it is easy to construct a bead that, if irradiated with light of a certain color $X$ (corresponding to the question: is your color $X$ ?), reacts illuminating itself with a light that corresponds to any of the colors of the rainbow with a pre-assigned probability (as already explained above, since this probability may be 0 or 1 , the exact case is included).

Even simpler would be to simulate this experiment on a computer.

If we introduce the rule (corresponding to Heisenberg principle) that two questions on different colors can never be posed simultaneously to the same bead, then no bead could ever be caught in contradiction.

Less obvious is the fact that the statistical behavior of the beads of type (II) can be quite different from those of type (I), that is from classical probability.

In order to illustrate this fact we consider the extreme case in which the beads have been programmed so as to answer Yes whichever the first question is. In such case, with the above stated rules, after the first answer they will have to confirm always the same color if asked the same question.

Experimentalist $A$, in order to estimate the fraction of red beads in the urn, extracts a certain number of beads (with re-insertion) and asks each of them the question Red?

With the above rules, the answer will always be: Yes .

$A$ is sure she is measuring a real property of the beads and, if she interprets the term reality in the passive sense and therefore the measurements as passive recordings of pre-existing properties (the interpretation underlying classical probability), then after a certain number of measurements she will conclude that all the beads in the box are red.

On the other hand experimentalist $B$ repeats the experiment asking the question Blue? and, with similar arguments, concludes that all the littles bead are blue.

No classic urn (i.e. with the colors of the bead fixed a priori independently of the question) could give rise to a result like the one just described. 
Therefore it is clear that, if one insists in identifying the notion of realism with that of passive realism (as it was implicitly done in the debate on the interpretation of QM), one will consider these experimental results as paradoxical.

In this extreme case, the only randomness is in the choice of the questions, but this is sufficient to illustrate the core of the statement: statistics of responses can give rise to countings of relative frequencies incompatible with every classical model.

The above example only deals with a deterministic situation, but we know from the theory of stochastic processes that a generic stochastic processes can always be written as a random perturbation of a deterministic system. Therefore, by introducing small random perturbations in a deterministic counter-example, one can easily produce proper statistical examples.

The extreme example, discussed above, can also illustrate the emerging of another important difference between the response and the passive recordings: non commutativity.

If we consider the question Red? as a black box that the bead crosses, coming out in the red state (similarly for Blue?), and if we denote (Blue?,Red?) the composite box, consisting in the fact that the bead is first asked the question Red? and then the question Blue?, then from this box the bead will come out in the Blue state, while from the composite box (Red? Blue?) it will come out in the Red state.

The properties of measurements are natural candidates for the extension of the notion of event because of the following reasons:

(i) include, as particular cases, the properties of the events

(ii) can give rise to non classical statistics

(iii) provide a natural and intuitive explanation of the emerging of non commutative operations in the description of the physical world

That is why one can obtain a unified axiomatic formulation of classic and quantum probability by enlarging the rigid notion of event into the more flexible notion of measurement.

The mathematical translation of the distinction between statistics of events (passive) and statistics of responses (adaptive) enters in the core of the debate on realism of the interpretation of quantum physics, that has been at the center of the philosophy of science in last the 70 years and that has seen scientists of the level Einstein and Schrödinger to accuse the standard interpretation of quantum theory not to respect the elementary standards of realism, and scientists of the level of Bohr and Heisenberg to argue on the impossibility of rendering such standards compatible with the results of the experiments.

The point of view of adaptive realism does not reject the ideas of the founding fathers of QT: it harmonizes them in a way that, up to a few years ago, would have seemed impossible and builds a unified picture. Bohr would accept the $Q P$ interpretation because it incorporates his main point: the active role of measurement. Heisenberg would accept it because it gives a more pragmatic and physically acceptable meaning to his idea of virtual reality. Einstein would like it because, as the EPR-chameleon experiment shows, it is perfectly compatible with pre-determination. Schrödinger would appreciate that, in this way, the interpretation of $Q T$ becomes free from those disturbing features which he didn't like.

Finally the intuitive justification, given above, of the emergence of non classical statistics allows to overcome Heisenberg's complaint that QM is "far from intuition": now we can explain to a housewife the conceptual difference between classical and quantum objects!

\section{REFERENCES}

1. L. Accardi: Stato fisico, Enciclopedia Einaudi Vol. 13 (1981) 514-548 (see also: Accardi L., Reviglio E.: The concept of physical state and the foundations of physics, unpublished)

2. Accardi L.: Foundations of Quantum Mechanics: a quantum probabilistic approach, in The Nature of Quantum Paradoxes ; eds. G.Tarozzi, A. van der Merwe Reidel (1988) 257-323, Preprint Dipartimento Di Matematica, Universita' di Roma Torvergata (1986)

3. The Växjö series of Conference Proceedings, 2000-2007 